\title{
A clinical study on stereotactic body radiotherapy for hepatocellular carcinoma with portal vein tumor thrombosis
}

\author{
Zhong NB${ }^{1}$, Chen $\mathrm{ZH}^{1}$ and $\mathrm{LV}_{\text {GM }}^{1, *}$ \\ ${ }^{1}$ Tumor Radiotherapy Center, Fuzhou General Hospital, Fuzhou, Fujian Provinve, 350025, China
}

\begin{abstract}
Purposes: Hepatocellular carcinoma with portal vein tumor thrombosis (HCC-PVTT) has a poor prognosis, while the optimal treatment remains controversial. The objective of this retrospective study was to evaluate the efficacy and safety of $\gamma$ - ray SBRT in treatment of patients with HCC-PVTT. Methods: The study was designed to examine the effects of $\gamma$-ray SBRT on toxicity and survival in patients with HCC-PVTT. To this end, data from patients with HCC-PVTT who received $\gamma$-ray SBRT during May 2008 to December 2011 was collected and analyzed. Response and (acute and late) toxicity were evaluated using the established international criteria. Log-rank test and Cox regression model were used to identify predictive factors and multivariate for survival, respectively. Results: The median follow up was 11 months. Fifty four consecutive patients with HCC-PVTT received daily fraction of 2.6 - 6Gy and six fractions per week for the total dose of 32.4 - 54Gy in 6 - 13 days. Six complete response (CR; 11.1\%) and twenty five partial response (PR; 46.3\%) were observed (overall response rate 57.4\%). Twenty three patients displayed a stable disease (SD; 42.6\%), while no patient experienced progressive disease (PD; 0\%). The treatment was well tolerated with no radiation-related complication and no $\geq$ Grade 3 toxicity. One- and twoyear overall survival rate were $33.4 \%$ and $13.6 \%$ respectively, and median overall survival was 10.7 months. The prognostic factors for survival included ECOG performance status $(\mathrm{P}=0.04)$, Child-Pugh score $(\mathrm{P}=0.05)$, PVTT size $(\mathrm{P}=0.02)$ and location $(\mathrm{P}=0.05)$. Conclusions: Individual $\gamma$ - ray SBRT appears to be feasible for treatment of patients with HCC-PVTT in whom other current therapies are contraindicated. PVTT size and ECOG performance status may represent the strongest predictive factors for survival.
\end{abstract}

Keywords: stereotactic body radiotherapy; portal vein tumor thrombosis; hepatocellular carcinoma; retrospective study

\section{Introduction}

Hepatocellular carcinoma (HCC) is one of the most common cancers in the world [1]. The early years of autopsy data reveals that more than $40 \%$ of HCC patients were accompanied by portal vein tumor thrombosis (PVTT) [2, 3]. In recent years, the reported incidence of PVTT was $44-62.8 \%$ in all HCC patients according to the autopsy data [4] and 31.4-34\% according to the clinical data [5]. PVTT leads to extremely poor prognosis of HCC patients, generally because first, pervasion of tumor cells via the portal vein leads to extensive bilobar intrahepatic metastases; second, obstruction of portal vein by PVTT results in deterioration in liver function or even liver failure; last, portal hypertension due to PVTT causes intractable ascites and esophageal variceal bleeding $[6,7]$. Median survival time of patients with HCCPVTT is less than three months without any treatment $[8,9]$. Despite the various existing therapies, such as hepatic resection, liver transplantation, radiotherapy, transarterial chemoembolization (TACE), and ablation therapy, the optimal regimen remains controversial $[10$,
11]. Therefore, an effective treatment for HCC-PVTT is desperately needed.

SBRT is a non-surgical procedure that delivers preciselytargeted radiation at much higher doses, in less fractions, compared to conventional radiation therapy. The major advantage is the radiobiological efficacy of such high fraction dose on tumor tissues, the short treatment course with a limited number of fractions and the non-

*Corresponding author: LV GM, Tumor Radiotherapy Center of Fuzhou General Hospital, Fuzhou, Fujian Provinve, 350025, China, Tel.: +011-86591-83782036; Fax: +011-86-591-83755909; Email: brightlgming@ hotmail.com

Received 19 March 2013 Revised 10 June 2013 Accepted 19 June 2013 Published 26 June 2013

Citation: Zhong NB, Chen ZH,LVGM (2013) A clinical study on stereotactic body radiotherapy for hepatocellular carcinoma with portal vein tumor thrombosis. J Cancer Res Ther 1: 143-148. doi:10.14312/20524994.2013-22

Copyright: (C) 2013 Zhong NB, et al. This is an open-access article distributed under the terms of the Creative Commons Attribution License, which permits unrestricted use, distribution and reproduction in any medium, provided the original author and source are credited. 
invasive outpatient therapy [12]. SBRT is typically used to treat small, early-stage tumors of the lung, or isolated recurrences or metastases from various types of cancer in lung, liver, adrenal gland, spine, etc. Several retrospective studies have shown promising results of SBRT in treatment of primary HCC, however, there are only a few reports on treatment of HCC-PVTT using SBRT. First, in a phase I study of 31 patients with Child-Pugh A large (median volume $173 \mathrm{~mL}$ ) unresectable HCC (14 patients had PVTT), patients who received SBRT for a total dose of 24-54 Gy (median dose $36 \mathrm{~Gy}$ ) in 6 fractions had the median survival of 11.7 months (11.6 months for patients with PVTT and 17.2 months for patients with no PVTT), 1 year local control of $65 \%$, and overall survival rate of $48 \%$, while no radiation-induced liver disease (RILD) or treatment-related grade $4 / 5$ toxicity was seen within 3 months [13]. Notably, RILD was considered a treatment limiting toxicity of conventional radiation treatments in HCC. Second, in a retrospective study (the median follow up was 10.5 months) of 31 patients with HCC [14], 9 patients with PVTT received SBRT combined with TACE, while 22 patients with small $(\leq 5 \mathrm{~cm})$ non-resectable HCC were given SBRT only. The results revealed that the overall response rate for small HCC and advanced HCC with PVTT was $82.6 \%$ (CR: $26.1 \%$ and PR: $56.5 \%$ ) and $44.4 \%$ (CR: $11.1 \%$ and PR: $33.3 \%$ ), with the median survival of 12 and 8 months, respectively. It was also noted that no patient experienced Grade 4 toxicity.

Thus, it remains to be defined whether individual $\gamma$ - ray SBRT is feasible, safe and effective in treatment of patients with HCC-PVTT. In order to expand the use of SBRT as an effective therapy for patients with HCC-PVTT who have very poor prognosis, we evaluated the response rate, survival, toxicity, and potential predictive factors in 54 patients with HCC-PVTT treated with $\gamma$ - ray SBRT.

\section{Materials and methods}

\section{Patient eligibility}

In this retrospective study, data were collected from the Tumor Radiotherapy Center of Fuzhou General Hospital, Fujian, China. Total of 68 consecutive HCC-PVTT patients were treated with $\gamma$ - ray SBRT between May 2008 and December 2011. 54 Patients were included in this study after retrospective review with institutional review board approval. The criteria for patients to be included in the study were as follows: (a) 18 years or older; (b) unresectable HCC with tumor thrombosis in the main and/or first branch of the portal vein; (c) Child-Pugh Class A or B; (d) Eastern Cooperative Oncology Group (ECOG) performance score $0-2$; and (e) no other treatment modalities being indicated. All patients had contrast-enhanced computed tomography (CT), magnetic resonance imaging (MRI) and/or positron emission tomography (PET) of abdomen. Blood tests included hepatitis B surface antigen, antibodies to hepatitis C, serum alpha-fetoprotein (AFP), serum albumin, and total bilirubin. HCC was diagnosed by cytological/histological evidence, one radiological image showing characteristic features of HCC together with an elevated serum alphafetoprotein (AFP) level (>400 ng/ml), or at least 2 radiological images showing characteristic features of HCC. Written informed consent was obtained from all patients before treatment with $\gamma$ - ray SBRT.

\section{Treatment}

SBRT was administrated using the total body gamma ray stereotactic radiotherapy system (OUR company, Shenzhen, China). Briefly, patients were immobilized by vacuum bags and underwent CT scan in supine or prostrate position. The CT data were transferred to the SBRT Treatment Planning System (SGI, Southeast University, China). The body surface, tumor (including PVTT) contour, and some important normal tissues were reconstructed to display three-dimensional (3D) representation. The clinical target volume (CTV) is defined as a macroscopic volume of tumor that includes the PVTT with $0.5-1.0 \mathrm{~cm}$ margins. The planning target volume (PTV) was created by symmetrically expanding the CTV by $0.5 \mathrm{~cm}$. The position, number and size of focused fields were elaborately selected to enhance the dose for the PTV but minimize both the dose for normal tissues and irradiated volumes. The generated dosevolume histogram (DVH) and isodose curves were used to evaluate the treatment planning. Dose prescription depends on the isodose curves that encompass the PTV. The PTV should be at least encompassed by $50 \%$ isodose curve in a qualified SBRT treatment planning. Therefore, dose prescription was normalized at $50-80 \%$ isodose curve normally. The total dose of 32.4 - 54.0Gy were delivered in 6 - 13 days, with fractional dose of 2.6-6Gy. If the PTV was adjacent to duodenum, fractional dose and total dose were restricted comparatively lower at 2.6-3.0Gy and 32.4Gy-38.4Gy, otherwise, fractional dose and total dose were 3.1-6Gy and 39.3-54Gy respectively. All patients have one day of rest after every 6 consecutive fractions of treatment. Verification films were taken to verify the tumors localization and patients position before SBRT.

\section{Evaluation of response, survival, and toxicity}

Patients were assessed for complete blood counts (CBC) and liver function tests weekly during treatment. Tumor and PVTT response within RT field were based on CT and/ or MRI scans 4 to 6 weeks after completion of treatment and 1 to 3 months intervals thereafter. Complete response (CR) was defined as complete disappearance of tumor and PVTT, partial response (PR) as a more than $30 \%$ reduction in the greatest diameter of tumor and PVTT, progressive disease (PD) as a more than $20 \%$ increase in the greatest diameter of tumor and PVTT, and stable disease (SD) as neither PR nor PD criteria is met. CR or PR was defined as objective response. The overall response (OR) rate was calculated by summing the rates of CR and PR. Survival time was estimated from the start of SBRT to the date of death or the last follow-up.

Acute and late toxicities were respectively assessed using the National Cancer Institute (NCI) Common Toxicity 
Criteria Version 2.0 and the Late Radiation Morbidity Scoring Scheme of Radiotherapy Oncology Group/ European Organization for Research and Treatment of Cancer (RTOG/EORTC), respectively.

\section{Statistical analysis}

Overall survival (OS) rate was calculated by the KaplanMeier method. The log-rank test was used to identify the predictive factors for survival. For multivariate analysis to evaluate the relation between the OS and various parameters, the stepwise procedure was performed using the Cox regression model. Differences of $\mathrm{P}<0.05$ were considered as statistically significant. Statistical analyses were conducted using the SPSS 16.0 statistical software package.

\section{Results}

\section{Patient characteristics}

Characteristics of patients and PVTT are summarized in Table 1. The median age of patients was $54.6 \mathrm{yr}$ (range,

Table 1 Characteristics of patients and tumor

\begin{tabular}{|c|c|c|}
\hline Variables & Values & $\begin{array}{l}\text { No. of } \\
\text { patients (\%) }\end{array}$ \\
\hline \multicolumn{3}{|l|}{ Age (yr) } \\
\hline Range & $32-83$ & \\
\hline Median & 54.6 & \\
\hline \multicolumn{3}{|l|}{ Gender } \\
\hline Male & & $43(79.6)$ \\
\hline Female & & $11(20.4)$ \\
\hline \multirow[t]{2}{*}{ ECOG performance status } & $0-1$ & $44(81.5)$ \\
\hline & 2 & $10(18.5)$ \\
\hline \multirow[t]{2}{*}{$\mathrm{AFP}(\mathrm{ng} / \mathrm{ml})$} & $\geq 400$ & 49 (90.7) \\
\hline & $<400$ & $5(9.3)$ \\
\hline \multirow[t]{2}{*}{ Child-Pugh class } & A & $47(87.0)$ \\
\hline & B & $7(13.0)$ \\
\hline \multirow[t]{5}{*}{ Location of PVTT } & Left branch & $17(31.5)$ \\
\hline & Right branch & $16(29.6)$ \\
\hline & Main+left branch & $9(16.7)$ \\
\hline & $\begin{array}{l}\text { Main+right } \\
\text { branch }\end{array}$ & $10(18.5)$ \\
\hline & $\begin{array}{l}\text { Main+left+right } \\
\text { branches }\end{array}$ & $2(3.7)$ \\
\hline \multirow[t]{2}{*}{ Tumor+PVTT size (mm) } & $\geq 50$ & $18(33.3)$ \\
\hline & $<50$ & $36(66.7)$ \\
\hline \multirow[t]{2}{*}{$\mathrm{C} / \mathrm{h}$ confirmation } & Yes & $43(79.6)$ \\
\hline & No & $11(20.4)$ \\
\hline \multirow[t]{2}{*}{ Metastasis } & Yes & $16(29.6)$ \\
\hline & No & $38(70.4)$ \\
\hline \multirow[t]{2}{*}{ Previous radiotherapy } & 0 & $46(85.2)$ \\
\hline & $\geq 1$ & $8(14.8)$ \\
\hline
\end{tabular}

Abbreviations: ECOG= Eastern Cooperative Oncology Group; AFP= alpha-fetoprotein; PVTT = portal vein tumor thrombosis; $\mathrm{c} / \mathrm{h}=$ cytological/histological.
32-82 yr). Among the patients, 43 (79.6\%) were male, and the male-to-female ratio was 3.9:1. The median size of tumor plus PVTT was $4.8 \mathrm{~cm}$ (range, $2-11 \mathrm{~cm}$ ). Serum hepatitis B virus antigen markers were positive in 36 patients $(66.7 \%)$ and hepatitis $C$ virus antigen in 5 patients $(9.3 \%)$. Specific sites of PVTT were as follows: left branch ( $\mathrm{n}=17,31.5 \%)$, right branch $(\mathrm{n}=16,29.6 \%)$, main portal vein + left branch $(n=9,16.7 \%)$, main portal vein + right branch $(\mathrm{n}=10,18.5 \%)$, and main portal vein + both left and right branches $(\mathrm{n}=2,3.7 \%)$.

All eligible patients had Child-Pugh classification A $(87.0 \%)$ or B (13.0\%). Ten patients $(18.5 \%)$ presented poor performance status (ECOG 2). 8 patients (14.8\%) had received previous radiotherapy (not including SBRT), and 16 patients (29.6\%) had intra- and/or extra-hepatic metastasis.

\section{PVTT response}

Among 54 patients, CR was achieved in 6 patients (11.1\%) and PR in 25 patients (46.3\%), with overall objective response rate of $57.4 \%$ (Table 2). 23 patients (42.6\%) displayed SD, while no PD was observed. Figure 1 shows the CT images before and after SBRT for a representative patient who was classified as a CR, in which no tumor recurrence was observed within the irradiated field in the liver after 13 months. Although tumors appeared in the livers of 19 patients during $0.4-2.1$ years after completion of $\gamma$ - ray SBRT, all newly-appeared tumors were found outside the irradiated fields.

Table 2 Response of HCC-PVTT treated with $\gamma$ - ray SBRT

\begin{tabular}{lc}
\hline Types of response & No. of patients (\%) \\
\hline Complete response (CR) & $6(11.1)$ \\
Partial response (PR) & $25(46.3)$ \\
Stable Disease (SD) & $23(42.6)$ \\
Overall response (OR = CR+PR) & $31(57.4)$ \\
\hline
\end{tabular}

\section{Survival outcomes}

The follow-up period ranged from 3 to 37 months (median 11 months) and 11 of 54 (20.4\%) patients were still alive at the time of last follow-up. 2 patients lost contact. Kaplan-Meier survival analysis indicated that the median survival was 10.7 months (range 6.3 to 34.4 months, Figure 2). The actuarial 1-year and 2-year overall survival rates assessed by the Life Tables Survival Analysis were $33.4 \%$ and $13.6 \%$, respectively.

The median survival in the patients with tumor plus PVTT size $<50 \mathrm{~mm}$ or $\geq 50 \mathrm{~mm}$ was 11.9 and 9.6 months, respectively. The patients with tumor plus PVTT size $<50 \mathrm{~mm}$ had a significantly higher median survival (P $=0.02$, Figure 3). A Cox regression analysis was then performed to determine the prognostic factors for 


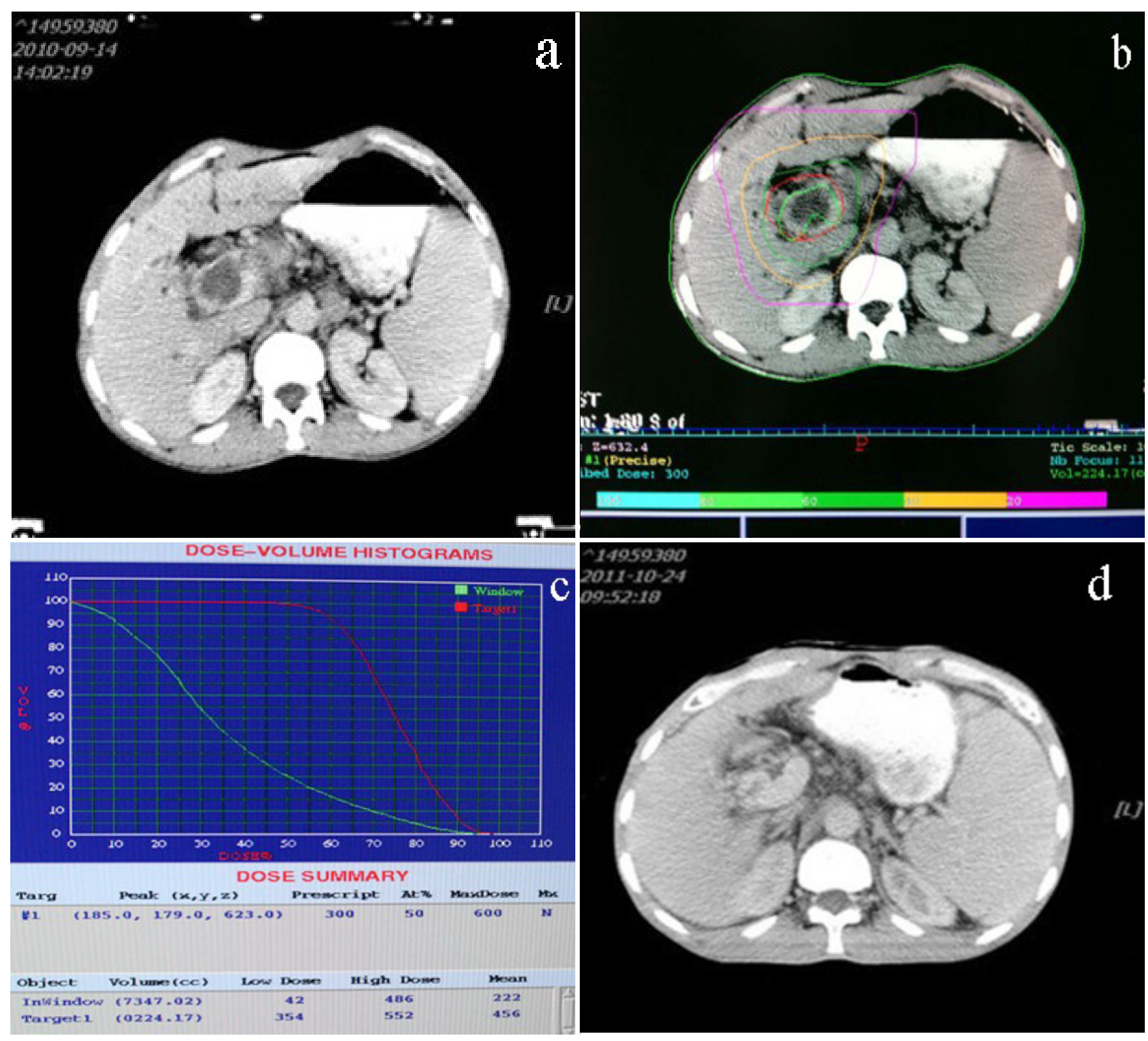

Figure 1 The abdominal CT scan before and after SBRT in a HCC-PVTT patient with a CR. A male patient with HCC-PVTT, who received $\gamma$ - ray SBRT with the dose of $3 \mathrm{~Gy} / \mathrm{F} \times 12 \mathrm{~F} / 2 \mathrm{w}$, achieved complete response. (a) CT scan before SBRT; (b and c) Isodose curve of target and dose-volume histogram generated by the treatment planning during treatment; (d) CT scan 1.1 years after SBRT, in which no PVTT was observed.

survival. The results indicated the potential prognostic factors for survival included ECOG performance status (0-1 vs. $2,13.1$ vs. 7.9 months, $P=0.04$ ), Child-Pugh score (A vs B, 11.7 vs. 9.8 months, $\mathrm{P}=0.05$ ), PVTT location (left or right branch only vs. main PV plus at least one of either left or right branch, 13.2 vs. 8.4 months, $\mathrm{P}=0.05$ ), lesion size $(<50 \mathrm{~mm}$ vs. $<50 \mathrm{~mm}, 11.9$ vs.9.6 months, $\mathrm{P}=0.02)$.

\section{Toxicity}

Overall, treatment was well tolerated, with no radiationinduced liver disease (RILD) or late complications observed during the median eleven-month follow-up period (3 - 37 months) after SBRT. Grade 1-2 acute gastrointestinal toxicity (e.g., appetite loss, vomiting, and diarrhea) were observed in 12 (22.2\%) patients.

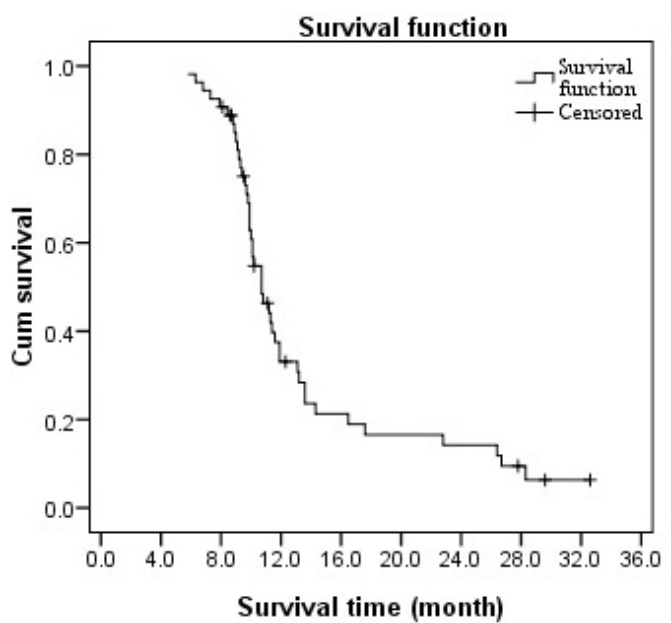

Figure 2 Overall survival of all patients. Kaplan-Meier curve for overall survival rate of 54 patients with HCC-PVTT treated with $\gamma$ - ray SBRT. 


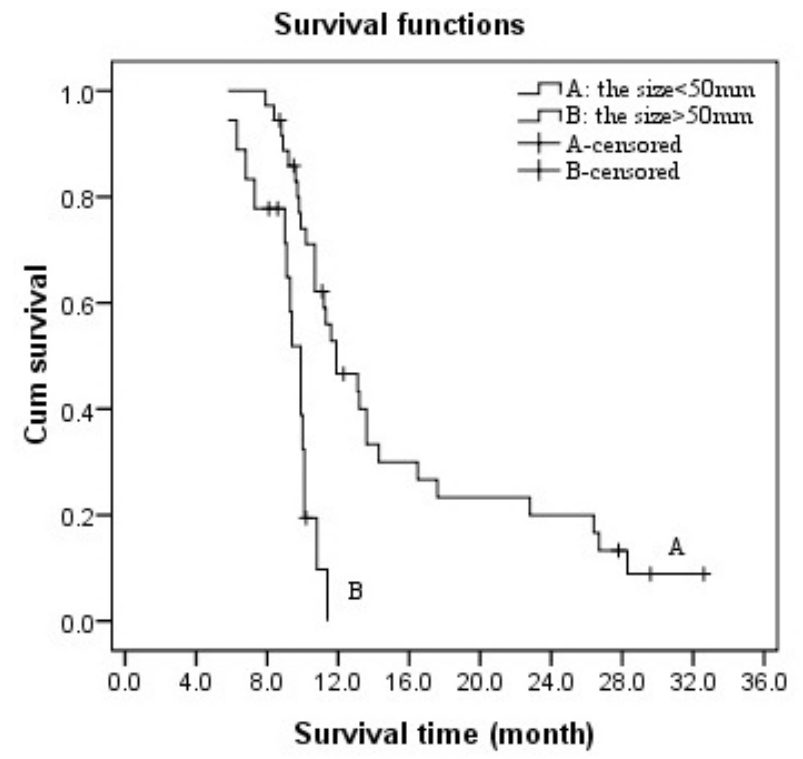

Figure 3 Effect of lesion size on survival. Overall survival curve according to the tumor + PVTT size $(A,<50 \mathrm{~mm} ; \mathrm{B}, \geq 50 \mathrm{~mm})$.

\section{Discussion}

HCC complicated with PVTT indicates that the disease is in an advanced stage [15]. The presence of PVTT is an extremely poor factor for prognosis of HCC [16]. It also represents an obstacle to the currently available therapies $[17,18]$. For example, surgical resection is one of the options to treat HCC-PVTT patients [19]. Minagawa et al reported the 5-year survival of HCC-PVTT patients after surgery was $10-30 \%$ [20]. However, only highly-selected patients are eligible for surgery [21-23]. Most patients are not suitable for surgical therapy due to the risk of post-operative liver failure and the early recurrence of disease. Another therapy for patients with HCC-PVTT is TACE $[24,25]$, while there is a little effect on PVTT and thus outcomes are usually not satisfied [26-28].

HCC used to be considered radioresistant. However, recent clinical studies indicate that HCC is actually a radiosensitive type of cancer $[29,30]$. Radiotherapy is also being used to treat HCC-PVTT [31-34], and has exhibited promising results. The most important issue in radiotherapy for HCC-PVTT is to keep normal liver tissue not to be irradiated, which would otherwise lead to liver failure particularly in HCC patients with poor liver function. SBRT is a radiotherapy that delivers preciselytargeted radiation at much higher doses on tumor tissues. The article by Bujold et al. [35] adds the evidence to the theory that SBRT represents an optimal treatment for HCC-PVTT.

In the current study, the response rate, overall survival rate, prognostic factors for survival and toxicity of 54 HCCPVTT patients treated with SBRT were retrospectively analyzed. Although the median survival in this study (10.7months) was shorter than HCC-PVTT treated with transplantation [36], all the outcomes were comparable with other (including surgery) approaches [37-39].
Importantly, there are no progressive disease and severe toxicity observed in our study. The reason might be that the advantage of $\gamma$-ray SBRT can easily meet the requirement of keeping enough normal liver not to be irradiated. Because 30 beams of $\gamma$-ray are all focused on the tumor tissue, this leading to increase dose on target but sparing normal liver tissue.

In conventional radiotherapy, patients generally receive the treatment in 35 to 49 days (5-7 weeks) and 5 consecutive fractions per week with daily fraction of 2 Gy. Because the longer treatment period is, the more dose loses. Toya [31] thus suggested that it should be more appropriate for HCC-PVTT patients treated with radiotherapy in shorter period. For SBRT treatment in the present study, all patients received the treatment in 6 to 13 days with daily fraction of $2.6-6 \mathrm{~Gy}$ and 6 fractions per week. Whereas fractional dose was higher than the conventional radiotherapy, the treatment period was also much shorter. However, there are certain limitations in this study due to the nature of a retrospective study. For example, the schedules of treatment were mainly determined by progression of the disease. Additionally, the relation between the dose and the liver volume did not study in this work also. Therefore, the well-designed prospective studies might be required to define the role of individual SBRT in treatment of HCC-PVTT.

\section{Conclusions}

$\gamma$-ray SBRT may be a safe and effective therapy in patients with HCC-PVTT who are contraindicated to other treatment modalities. It warrants further attention in HCC-PVTT.

\section{Conflict of interest}

All the authors declare that they have no conflict of interest.

\section{References}

[1] Cormier JN, Thomas KT, Chari RS, Pinson CW (2006) Management of hepatocellular carcinoma. J Gastrointest Surg 10: 761-780.

[2] Pirisi M, Avellini C, Fabris C, Scott C, Bardus P, et al. (1998) Portal vein thrombosis in hepatocellular carcinoma: age and sex distribution in an autopsy study. J Cancer Res Clin Oncol 124: 397-400.

[3] Nakashima T, Okuda K, Kojiro M, Jimi A, Yamaguchi R, et al. (1983) Pathology of hepatocellular carcinoma in Japan. 232 Consecutive cases autopsied in ten years. Cancer 51: 863-877.

[4] Ikai I, Arii S, Okazaki M, Okita K, Omata M, et al. (2007) Report of the 17th Nationwide Follow-up Survey of Primary Liver Cancer in Japan. Hepatol Res 37: 676-691.

[5] Park KW, Park JW, Choi JI, Kim TH, Kim SH, et al. (2008) Survival analysis of 904 patients with hepatocellular carcinoma in a hepatitis B virus-endemic area. J Gastroenterol Hepatol 23: 467473.

[6] Lau WY (2002) Management of hepatocellular carcinoma. J R Coll Surg Edinb 47: 389-399.

[7] Lai EC, Lau WY (2005) The continuing challenge of hepatic cancer in Asia. Surgeon 3: 210-215. 
[8] Llovet JM, Bustamante J, Castells A, Vilana R, Ayuso Mdel C, et al. (1999) Natural history of untreated nonsurgical hepatocellular carcinoma: rationale for the design and evaluation of therapeutic trials. Hepatology 29: 62-67.

[9] Villa E, Moles A, Ferretti I, Buttafoco P, Grottola A, et al. (2000) Natural history of inoperable hepatocellular carcinoma: estrogen receptors' status in the tumor is the strongest prognostic factor for survival. Hepatology 32: 233-238.

[10] Lau WY, Lai EC and Yu SC. (2008) Hepatocellular Carcinoma. World Scientific Publishing Co Pte Ltd, Singapore.

[11] Shuqun C, Mengchao W, Han C, Feng S, Jiahe Y, et al. (2007) Tumor thrombus types influence the prognosis of hepatocellular carcinoma with the tumor thrombi in the portal vein. Hepatogastroenterology 54: 499-502.

[12] Ursino S, Greco C, Cartei F, Colosimo C, Stefanelli A, et al. (2012) Radiotherapy and hepatocellular carcinoma: update and review of the literature. Eur Rev Med Pharmacol Sci 16: 1599-1604.

[13] Tse RV, Hawkins M, Lockwood G, Kim JJ, Cummings B, et al. (2008) Phase I study of individualized stereotactic body radiotherapy for hepatocellular carcinoma and intrahepatic cholangiocarcinoma. J Clin Oncol 26: 657-664.

[14] Choi BO, Choi IB, Jang HS, Kang YN, Jang JS, et al. (2008) Stereotactic body radiation therapy with or without transarterial chemoembolization for patients with primary hepatocellular carcinoma: preliminary analysis. BMC Cancer 8: 351 .

[15] Bruix J, Sherman M (2005) Management of hepatocellular carcinoma. Hepatology 42: 1208-1236.

[16 ] Giannelli G, Pierri F, Trerotoli P, Marinosci F, Serio G, et al. (2002) Occurrence of portal vein tumor thrombus in hepatocellular carcinoma affects prognosis and survival. A retrospective clinical study of 150 cases. Hepatol Res 24: 50-59.

[17] Urata K, Matsumata T, Kamakura T, Hasuo K, Sugimachi K (1994) Lipiodolization for unresectable hepatocellular carcinoma: an analysis of 205 patients using univariate and multivariate analysis. J Surg Oncol 56: 54-58.

[18] Fujii T, Takayasu K, Muramatsu Y, Moriyama N, Wakao F, et al. (1993) Hepatocellular carcinoma with portal tumor thrombus: analysis of factors determining prognosis. Jpn J Clin Oncol 23: 105-109.

[19] Poon D, Anderson BO, Chen LT, Tanaka K, Lau WY, et al. (2009) Management of hepatocellular carcinoma in Asia: consensus statement from the Asian Oncology Summit 2009. Lancet Oncol 10: 1111-11118.

[20] Minagawa M, Makuuchi M (2006) Treatment of hepatocellular carcinoma accompanied by portal vein tumor thrombus. World J Gastroenterol 12: 7561-7567.

[21] Shi J, Lai EC, Li N, Guo WX, Xue J, et al. (2010) Surgical treatment of hepatocellular carcinoma with portal vein tumor thrombus. Ann Surg Oncol 17 : 2073-2080.

[22] Yoshidome H, Takeuchi D, Kimura F, Shimizu H, Ohtsuka M, et al. (2011) Treatment strategy for hepatocellular carcinoma with major portal vein or inferior vena cava invasion: a single institution experience. J Am Coll Surg 212 : 796-803.

[23] Chirica M, Scatton O, Massault PP, Aloia T, Randone B, et al. (2008) Treatment of stage IVA hepatocellular carcinoma: should we reappraise the role of surgery? Arch Surg 143: 538-543.

[24] Uraki J, Yamakado K, Nakatsuka A, Takeda K (2004) Transcatheter hepatic arterial chemoembolization for hepatocellular carcinoma invading the portal veins: therapeutic effects and prognostic factors. Eur J Radiol 51: 12-18.

[25] Meng MB, Cui YL, Lu Y, She B, Chen Y, et al. (2009) Transcatheter arterial chemoembolization in combination with radiotherapy for unresectable hepatocellular carcinoma: a systematic review and meta-analysis. Radiother Oncol 92: 184-194.

[26] Ishikura S, Ogino T, Furuse J, Satake M, Baba S, et al. (2002) Radiotherapy after transcatheter arterial chemoembolization for patients with hepatocellular carcinoma and portal vein tumor thrombus. Am J Clin Oncol 25: 189-193.
[27] Ando E, Tanaka M, Yamashita F, Kuromatsu R, Yutani S, et al. (2002) Hepatic arterial infusion chemotherapy for advanced hepatocellular carcinoma with portal vein tumor thrombosis: analysis of 48 cases. Cancer 95: 588-595.

[28] Yen FS, Wu JC, Kuo BI, Chiang JH, Chen TZ, et al. (1995) Transcatheter arterial embolization for hepatocellular carcinoma with portal vein thrombosis. J Gastroenterol Hepatol 10: 237240.

[29] Zeng ZC, Jiang GL, Wang GM, Tang ZY, Curran WJ, et al. (2002) DNA-PKcs subunits in radiosensitization by hyperthermia on hepatocellular carcinoma hepG2 cell line. World J Gastroenterol 8: 797-803.

[30] Hawkins MA, Dawson LA (2006) Radiation therapy for hepatocellular carcinoma: from palliation to cure. Cancer 106: 1653-1663.

[31] Toya R, Murakami R, Baba Y, Nishimura R, Morishita S, et al. (2007) Conformal radiation therapy for portal vein tumor thrombosis of hepatocellular carcinoma. Radiother Oncol 84: 266-271.

[32] Huang YJ, Hsu HC, Wang CY, Wang CJ, Chen HC, et al. (2009) The treatment responses in cases of radiation therapy to portal vein thrombosis in advanced hepatocellular carcinoma. Int J Radiat Oncol Biol Phys 4: 1155-1163.

[33] Zeng ZC, Fan J, Tang ZY, Zhou J, Qin LX, et al. (2005) A comparison of treatment combinations with and without radiotherapy for hepatocellular carcinoma with portal vein and/or inferior vena cava tumor thrombus. Int J Radiat Oncol Biol Phys 61: 432-443.

[34] Zhang XB, Wang JH, Yan ZP, Qian S, Du SS, et al. (2009) Hepatocellular carcinoma with main portal vein tumor thrombus: treatment with 3-dimensional conformal radiotherapy after portal vein stenting and transarterial chemoembolization. Cancer 115: 1245-1252.

[35] Bujold A, Massey CA, Kim JJ, Brierley J, Cho C, et al. (2013) Sequential phase I and II trials of stereotactic body radiotherapy for locally advanced hepatocellular carcinoma. J Clin Oncol 31: 1631-1639.

[36] Connolly GC, Chen R, Hyrien O, Mantry P, Bozorgzadeh A, et al. (2008) Incidence, risk factors and consequences of portal vein and systemic thromboses in hepatocellular carcinoma. Thromb Res 122: 299-306.

[37] Peng BG, He Q Li JP, Zhou F (2009) Adjuvant transcatheter arterial chemoembolization improves efficacy of hepatectomy for patients with hepatocellular carcinoma and portal vein tumor thrombus. Am J Surg 198: 313-318.

[38] Pawlik TM, Poon RT, Abdalla EK, Ikai I, Nagorney DM, et al (2005) Hepatectomy for hepatocellular carcinoma with major portal or hepatic vein invasion: results of a multicenter study. Surgery 137 : 403-410.

[39] Chen JS, Wang Q Chen XL, Huang XH, Liang LJ, et al. (2012) Clinicopathologic characteristics and surgical outcomes of hepatocellular carcinoma with portal vein tumor thrombosis. J Surg Res 175: 243-250. 